\title{
Effects of grape seed extract supplementation on exercise-induced oxidative stress in rats
}

\author{
Muaz Belviranlı ${ }^{1 *}$, Hakkı Gökbel ${ }^{1}$, Nilsel Okudan ${ }^{1}$ and Kemal Başaralı ${ }^{2}$ \\ ${ }^{1}$ Department of Physiology, Meram Faculty of Medicine, Selcuk University, Konya, Turkey \\ ${ }^{2}$ Department of Biochemistry, Faculty of Medicine, Dicle University, Diyarbakir, Turkey \\ (Submitted 20 June 2011 - Final revision received 18 August 2011 - Accepted 8 September 2011 - First published online 20 October 2011)
}

\section{Abstract}

The aim of the present study was to investigate the effects of grape seed extract (GSE) supplementation on exercise performance and oxidative stress in acutely and chronically exercised rats. A total of sixty-four male rats were used in the study. Rats were divided into six groups: control, chronic exercise control, acute exercise control (AEC), GSE-supplemented control, GSE-supplemented chronic exercise and GSE-supplemented acute exercise groups. Chronic exercise consisted of treadmill running at $25 \mathrm{~m} / \mathrm{min}, 45 \mathrm{~min} / \mathrm{d}$, $5 \mathrm{~d}$ a week for 6 weeks. Rats in the acute exercise groups were run on the treadmill at $30 \mathrm{~m} / \mathrm{min}$ until exhaustion. GSE were given at $100 \mathrm{mg} / \mathrm{kg}$ of body weight with drinking water for 6 weeks. Plasma was separated from blood samples for the analysis of oxidative stress markers. There was no significant difference in time of exhaustion between the acute exercise groups. Plasma malondialdehyde (MDA) levels were higher in the acute exercise groups and lower in the chronic exercise groups. GSE supplementation decreased MDA levels. Xanthine oxidase and adenosine deaminase activities were higher in the AEC group compared to all the other groups. NO levels were increased with both chronic exercise and GSE supplementation. Superoxide dismutase and glutathione peroxidase activities were lower in the acute exercised groups and higher in the chronic exercised groups. GSE supplementation caused an increase in antioxidant enzyme activities. In conclusion, GSE supplementation prevents exercise-induced oxidative stress by preventing lipid peroxidation and increasing antioxidant enzyme activities.

Key words: Grape seed extract: Exercise: Oxidative stress

Exercise increases the utilisation of oxygen in the body, and therefore enhances the production of reactive oxygen species (ROS) and impairs both enzymatic and non-enzymatic antioxidant defence systems in target tissues and blood ${ }^{(1,2)}$. An increase in ROS production may occur during and after exercise by increase in oxygen uptake, increase in catecholamine levels, increase in lactic acid production, elevated rate of $\mathrm{Hb}$ auto-oxidation and hyperthermia ${ }^{(3)}$. As a consequence of increased production of ROS, oxidative damage of lipids, proteins and DNA has been reported following single bouts of exercise ${ }^{(1,4,5)}$. However, it is generally accepted that regular, non-exhaustive exercise training reduces post-exercise oxidative stress in blood and tissues ${ }^{(6)}$.

The increase in ROS production is usually protected by antioxidant defence systems, such as antioxidant enzymes, non-enzymatic antioxidant mechanism and antioxidant vitamins ${ }^{(7)}$. Antioxidant levels are influenced by nutritional, pathological and physiological factors and their efficiency in counterbalancing ROS production determines the level of cell damage ${ }^{(3)}$. Exercise-induced oxidative damage may be prevented by optimising nutrition, particularly by increasing the dietary content of nutritional antioxidants ${ }^{(8)}$. Supplementation of certain antioxidant nutrients is practicable to recover faster from tiredness and prevent exercise-induced oxidative damage $^{(9)}$. Many studies ${ }^{(10,11)}$ have indicated that antioxidant supplementation led to the prevention of strenuous exerciseinduced oxidative injury in rats.

Grape seed extract (GSE) contains plant flavonoids such as proanthocyanidins. Flavonoids are potent antioxidants and exert many health-promoting effects ${ }^{(12)}$. The antioxidant effect of GSE is approximately fifty times greater than that of vitamin $\mathrm{C}$ and vitamin $\mathrm{E}^{(13)}$. There are limited studies ${ }^{(14,15)}$ investigating the effects of grape or grape leaf extract on exercise performance or exercise-induced oxidative stress, but no study to date has investigated the effects of GSE

\footnotetext{
Abbreviations: ADA, adenosine deaminase; AEC, acute exercise control; C, control; CEC, chronic exercise control; GAE, grape seed extract-supplemented acute exercise; GC, grape seed extract-supplemented control; GCE, grape seed extract-supplemented chronic exercise; GPx, glutathione peroxidase; GSE, grape seed extract; MDA, malondialdehyde; ROS, reactive oxygen species; SOD, superoxide dismutase; XO, xanthine oxidase.
} 
supplementation on exercise-induced oxidative stress in acutely and/or chronically exercised rats.

In the present study, we aimed to investigate changes in blood because it has been suggested that oxidative stress markers change towards the same direction in blood and tissues; and this implies that changes detected in blood markers may reflect changes in the redox status of tissues such as skeletal muscle, heart or liver ${ }^{(16)}$. Since the antioxidant properties of GSE have been shown in previous studies ${ }^{(17-21)}$ and free radicals mediate exercise-induced oxidative stress, we designed the present study based on the effects of GSE supplementation on exercise-induced oxidative damage in acutely and chronically exercised rats.

\section{Materials and methods}

\section{Animals}

The experiments were carried out with sixty-four adult male Sprague-Dawley rats (14 weeks of age, weighing 243.4 (SD $29 \cdot 2) \mathrm{g}$ ). Rats were housed under controlled environmental conditions $\left(12 \mathrm{~h}\right.$ light $-12 \mathrm{~h}$ dark cycle and $21 \pm 2^{\circ} \mathrm{C}$ temperature and $50 \%$ humidity). The rats were fed ad libitum a standard rat chow and tap water. The body weight changes of the animals were recorded weekly. The study protocol was approved by the Ethics Committee of the Selcuk University Experimental Medicine Research and Application Center (Konya, Turkey). All procedures were in accordance with the US National Institutes of Health Guide for the Care and Use of Laboratory Animals.

Rats were randomly divided into the following six groups: sedentary control (C, $n$ 10), chronic exercise control (CEC, $n$ 11), acute exercise control (AEC, $n$ 11), GSE-supplemented control (GC, $n$ 10), GSE-supplemented chronic exercise (GCE, $n$ 11) and GSE-supplemented acute exercise (GAE, $n$ 11).

\section{Supplementation protocol}

GSE was kindly donated by San Joaquin Valley Concentrates (Fresno, CA, USA). GSE was extracted with a standardised water-ethanol mixture, and in previous studies ${ }^{(18,22)}$ it had been demonstrated that GSE contains dimeric (54\%), trimeric (13\%), tetrameric (7\%) and monomeric proanthocyanidines (less than 5\%). Antioxidants such as cathechines and oligomeric proanthocyanidines also exist in the extract.

Rats in the GC, GCE and GAE groups were given GSE in drinking-water at $100 \mathrm{mg} / \mathrm{kg}$ per $\mathrm{d}$ for 6 weeks and this GSE concentration in drinking-water corresponds to approximately $100 \mathrm{mg} / \mathrm{kg}$ per $\mathrm{d}$. The $100 \mathrm{mg} / \mathrm{kg}$ dosage was chosen because previous studies ${ }^{(23,24)}$ have demonstrated the antioxidant effect of this dosage in rats.

\section{Exercise protocol}

The exercise protocols were performed on a motor-driven rodent treadmill (MAY-TME 0804, Commat Limited, Ankara, Turkey). The treadmill was equipped with an electric shock grid on the rear barrier to provide exercise motivation to the animals. All exercise tests were performed during the same time period of the day to minimise diurnal effects.

The animals in the chronic exercise groups (CEC and GCE groups) were habituated by treadmill exercise over a 5-d period such as: 1 st day $10 \mathrm{~m} / \mathrm{min}, 10 \mathrm{~min}$; 2 nd day $20 \mathrm{~m} / \mathrm{min}$; $10 \mathrm{~min}$, 3rd day $25 \mathrm{~m} / \mathrm{min}, 10 \mathrm{~min}$; 4th day $25 \mathrm{~m} / \mathrm{min}, 20 \mathrm{~min}$ and 5 th day $25 \mathrm{~m} / \mathrm{min}, 30 \mathrm{~min}$. Thereafter, the animals were exercised at $25 \mathrm{~m} / \mathrm{min}, 45 \mathrm{~min} / \mathrm{d}, 5 \mathrm{~d}$ per week for 6 weeks ${ }^{(25)}$.

The animals in the acute exercise groups underwent a light exercise familiarisation on the treadmill $3 \mathrm{~d}$ before the exercise protocol at $15 \mathrm{~m} / \mathrm{min}$ for $15 \mathrm{~min}$. Immediately before being killed, the rats were made to run on the treadmill at $30 \mathrm{~m} / \mathrm{min}$ until exhaustion and time to exhaustion was recorded ${ }^{(26)}$. Exhaustion was defined as the inability of a rat to right itself when being laid on its side. To minimise diurnal effects, all animals were exercised at the same time (09.00-12.00 hours).

\section{Blood sampling}

The rats were killed immediately post-exercise in the acute exercise group and $24 \mathrm{~h}$ after the last exercise in the chronic exercise group (to wean the effects of acute exercise) by cardiac puncture. To minimise diurnal effects, all animals were killed at the same hours. Within $1 \mathrm{~min}$, blood samples were transferred into EDTA-coated tubes and plasma was separated by centrifugation at $1750 \mathrm{~g}$ for $10 \mathrm{~min}$ at $+4^{\circ} \mathrm{C}$. Plasma samples were stored at $-80^{\circ} \mathrm{C}$ until the time of analysis.

\section{Biochemical analysis}

The plasma malondialdehyde (MDA) levels were measured by the thiobarbituric acid-reactive substances assay according to the method of Wasowicz et al. ${ }^{(27)}$. The quantification of thiobarbituric acid-reactive substances was determined by comparing the absorption to the standard curve of MDA equivalents generated by acid catalysed hydrolysis of 1,1,3,3 tetramethoxypropane. The values of MDA were expressed as $\mu \mathrm{mol} / 1$.

Plasma xanthine oxidase (XO) activity was determined by the method of Prajda \& Weber ${ }^{(28)}$, wherein activity is measured by the determination of uric acid from xanthine. The reaction was stopped by the addition of $100 \%$ (w/v) TCA. A calibration curve was constructed by using $10-50 \mathrm{mU} / \mathrm{ml}$ concentrations of standard XO solutions. Urate was determined in the supernatant by measuring the absorption at $293 \mathrm{~nm}$ against blank. One unit of activity was defined as $1 \mu \mathrm{mol}$ of uric acid formed per min at $37^{\circ} \mathrm{C}, \mathrm{pH} 7 \cdot 5$, and expressed in $\mathrm{U} / \mathrm{ml}$.

Plasma adenosine deaminase (ADA) activities were estimated spectrophotometrically by the method of Giusti ${ }^{(29)}$, which is based on the direct measurements of the formation of ammonia produced when ADA acts in excess of adenosine. The results of ADA were expressed as $\mathrm{U} / \mathrm{l}$.

Endogenous production of $\mathrm{NO}$ in plasma was determined as nitrite and nitrate. Nitrite and nitrate levels were determined in plasma by acidic Griess reaction using a spectrophotometric method after deproteinisation ${ }^{(30)}$. The values of NO were expressed as mmol/1. 
The plasma total superoxide dismutase (SOD) activity was measured using a commercially available SOD assay kit (Cayman Chemical, Ann Arbor, MI, USA) according to the manufacturer's instructions. This assay measures all three types of SOD iso-enzymes $(\mathrm{Cu} / \mathrm{Zn}, \mathrm{Mn}$ and FeSOD). The activity was recorded spectrophotometrically at $450 \mathrm{~nm}$. The enzyme activities were expressed as $\mathrm{U} / \mathrm{ml}$.

Plasma glutathione peroxidase (GPx) activity was measured using enzymatic procedures as described by the provider (Cayman Chemical). GPx activity was monitored spectrophotometrically at $340 \mathrm{~nm}$ for $5 \mathrm{~min}$. The decrease in absorbance at $340 \mathrm{~nm}$ is proportional to the GPx activity in the sample. Activity was expressed as $\mathrm{nmol} / \mathrm{min}$ per $\mathrm{ml}$ ).

\section{Statistical analysis}

Statistical analysis was carried out by using SPSS 15.0 for Windows (SPSS Inc., Chicago, IL, USA). All data are presented as means and standard deviations. All the data were tested for homogeneity of variance. The effects of the GSE supplementation on oxidative stress and antioxidant defence markers in acute and chronic exercise were tested by a two-way ANOVA, with GSE supplementation and the exercise tests as ANOVA factors. The sets of data in which there were significant effects were tested by the one-way ANOVA with Bonferroni correction and post hoc Tukey test. Exhaustion time of the acute exercise groups were compared with Student's $t$ test. A $P$ value less than 0.05 was considered statistically significant.

\section{Results}

\section{Effects of grape seed extract supplementation on time of exhaustion}

There was no difference in time of exhaustion between the acute exercised rats. GSE supplementation did not affect time of exhaustion in the acutely exercised rats (71.4 (SD 16.2) $v$. $70 \cdot 8$ (SD 13.3) $\mathrm{min}$ in the AEC and GAE groups, respectively).

\section{Effects of grape seed extract supplementation and acute and chronic exercise on malondialdehyde levels}

Plasma MDA levels $(\mu \mathrm{mol} / \mathrm{l})$ were higher in the acute exercised groups while significantly lower in the chronic exercised groups compared to the controls $(P<0.05)$. Plasma MDA levels were lower in all the GSE-supplemented groups compared to the non-supplemented controls (GC, GCE, GAE; 1.13 (sD 0.05), 0.83 (sD 0.06), 1.31 (sD 0.05) v. C, CEC, AEC; 1.29 (SD 0.05), 1.07 (SD 0.04), 1.54 (SD 0.08), respectively; $P<0.05)$. The highest MDA levels were observed in the AEC group and the lowest MDA levels were observed in the GCE group (Fig. 1).

\section{Effects of grape seed extract supplementation and acute and chronic exercise on xanthine oxidase activities}

Plasma XO activity $(\mathrm{U} / \mathrm{ml})$ was the highest in the acutely exercised control group compared to the other groups including

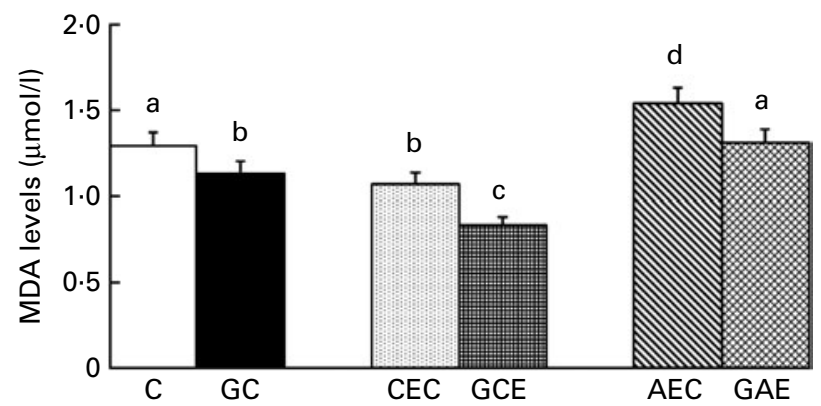

Fig. 1. Effects of acute or chronic exercise and grape seed extract (GSE) supplementation on plasma malondialdehyde (MDA) levels. ${ }^{a, b, c, d}$ Mean values with unlike letters were significantly different $(P<0.05$; two-way ANOVA). There was a significant exercise $\times$ GSE interaction $(P=0.045)$. C, control; GC, GSE-supplemented control; CEC, chronic exercise control GCE, GSE-supplemented chronic exercise; AEC, acute exercise control; GAE, GSE-supplemented acute exercise.

the GSE-supplemented acute exercise group (AEC and GAE groups; 0.41 (SD 0.05) and 0.27 (SD 0.04), respectively; $P<0 \cdot 05)$. There was no significant difference among the other groups ( $P>0 \cdot 05$; Fig. 2).

\section{Effects of grape seed extract supplementation and acute and chronic exercise on adenosine deaminase activities}

Plasma ADA activity (U/1) was the highest in the acutely exercised control group compared to all the other groups including the GSE-supplemented acute exercise group (AEC and GAE groups; 25.98 (SD 2.77) and 17.04 (SD 4.45), respectively; $P<0 \cdot 05)$. There was no significant difference among the other groups $(P>0 \cdot 05$; Fig. 3$)$.

\section{Effects of grape seed extract supplementation and acute and chronic exercise on NO levels}

Plasma NO levels $(\mathrm{mmol} / \mathrm{l})$ were higher in all the GSEsupplemented groups compared to the non-supplemented controls (GC, GCE, GAE; 94.22 (SD 7.77), 116.91 (SD 8.48), $100.90(\mathrm{SD} 13.69)$ v. C, CEC, AEC; 75.00 (SD 8.71), 95.15

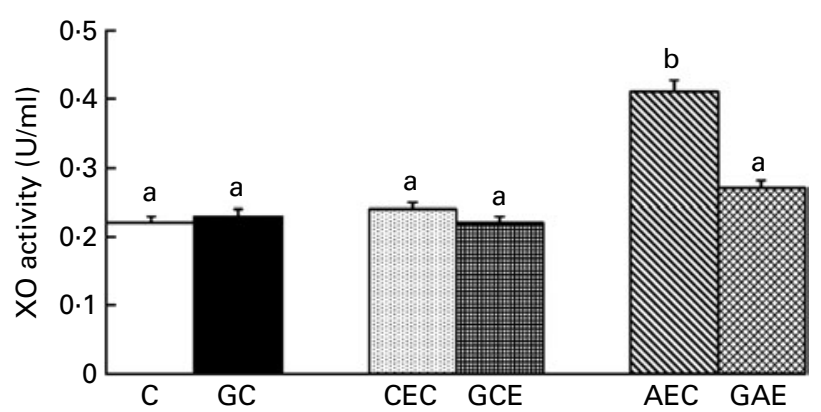

Fig. 2. Effects of acute or chronic exercise and grape seed extract (GSE) supplementation on plasma xanthine oxidase $(X O)$ activities. ${ }^{a, b}$ Mean values with unlike letters were significantly different $(P<0.05$; two-way ANOVA). There was a significant exercise $\times$ GSE interaction $(P=0.002)$. C, control; GC, GSE-supplemented control; CEC, chronic exercise control; GCE, GSEsupplemented chronic exercise; AEC, acute exercise control; GAE, GSEsupplemented acute exercise. 




Fig. 3. Effects of acute or chronic exercise and grape seed extract (GSE) supplementation on plasma adenosine deaminase (ADA) activities. ${ }^{a, b}$ Mean values with unlike letters were significantly different $(P<0.05$; two-way ANOVA). There was a significant exercise $\times$ GSE interaction $(P=0.000)$. C, control; GC, GSE-supplemented control; CEC, chronic exercise control; GCE, GSE-supplemented chronic exercise; AEC, acute exercise control; GAE, GSE-supplemented acute exercise.

(SD 6.10), $78 \cdot 77$ (SD 6.80), respectively; $P<0 \cdot 05$ ). There was no difference between the control and acute exercised groups $(P>0.05)$, but plasma NO levels were higher in the chronically exercised groups $(P<0 \cdot 05$; Fig. 4). The GCE group had the highest NO levels.

\section{Effects of grape seed extract supplementation and acute and chronic exercise on superoxide dismutase activities}

Plasma SOD activity (U/ml) was higher in all the GSEsupplemented groups compared to the non-supplemented controls (GC, GCE, GAE; 1.088 (SD 0.05), 1.419 (SD 0.04), 0.908 (SD 0.07) v. C, CEC, AEC; 0.903 (SD 0.05), 1.260 (SD 0.02 ), 0.704 (SD 0.03), respectively; $P<0.05$ ). Plasma SOD activity was lower in the acute exercised groups while it was higher in the chronic exercised groups compared to the controls $(P<0.05)$. The highest SOD activity was observed in the GCE group and the lowest SOD activity was observed in the AEC group (Fig. 5).

\section{Effects of grape seed extract supplementation and acute} and chronic exercise on glutathione peroxidase activities

Plasma GPx activity ( $\mathrm{nmol} / \mathrm{min}$ per $\mathrm{ml}$ ) was higher in both the acute and chronic exercised and the GSE-supplemented groups compared to the non-supplemented controls (GCE, GAE; 166.96 (SD 17.51), 98.01 (SD 5.99) v. CEC, AEC; 137.87 (SD 19.28), $77 \cdot 13$ (SD 5.67), respectively; $P<0.05$ ). Plasma GPx activity was lower in the acute exercised groups while it was significantly higher in the chronic exercised groups compared to the controls $(P<0 \cdot 05)$. There was no difference between the control groups (GC, C; 110.32 (SD 3.43), 99.19 (SD 7.28), respectively). The highest GPx activity was observed in the GCE group and the lowest GPx activity was observed in the AEC group (Fig. 6).

\section{Discussion}

In recent years, there has been a great deal of interest in the antioxidant potentials of phytochemicals such as green tea, ginseng and grape seed extract. The antioxidant effects of
GSE were demonstrated in many conditions such as chemical-induced oxidative stress $^{(17,18)}$, hypoxia ${ }^{(19)}$, X-radiation $^{(31)}$ and ischaemia-reperfusion injury ${ }^{(20,21)}$. In these studies, GSE supplementation reduced lipid peroxidation and played a protective role against increased oxidative damage in blood and tissues. However, no study to date has investigated the effects of GSE supplementation on oxidative stress markers in acutely and/or chronically exercised rats or human subjects. In the present study, we hypothesised that GSE supplementation would partially protect the antioxidant system and therefore alleviate acute and chronic exercise-induced oxidative damage.

In the present study, the exhaustion time of the acuteexercised GSE-supplemented group (GAE) was not different from that of the control (AEC). Based on these findings, we suggest that GSE supplementation does not influence the performance of exhaustive exercise in rats. In the present literature, there is no study investigating the effects of GSE supplementation on exercise performance. However, Lafay et $a l .{ }^{(15)}$ demonstrated that grape extract supplementation increases physical performance and explosive power in elite athletes during competition period (handball players). Further and more detailed studies are needed in this subject.

This study compared the changes in several oxidative stress biomarkers, including MDA, XO, ADA, NO, SOD and GPX in blood samples in response to acute and chronic exercise and dietary antioxidant pretreatment (GSE). The present study has demonstrated that while acute exhaustive exercise induced oxidative stress and impaired antioxidant enzyme activities, 6 weeks of exercise training attenuated oxidative stress and improved antioxidant enzyme activities in blood. Furthermore, GSE supplementation reduced exercise-induced oxidative damage and augmented the activity of antioxidant enzymes in plasma.

The data presented here show that while an acute exhaustive exercise protocol led to increased MDA levels in plasma, 6 weeks of exercise training led to a reduction in MDA levels and to a protection of cells and tissues against oxidative damage. Furthermore, GSE supplementation reduced MDA levels in all groups and played a protective role against increased oxidative damage in blood. It has been demonstrated that green tea polyphenols reduced post-exercise lipid peroxidation in



Fig. 4. Effects of acute or chronic exercise and grape seed extract (GSE) supplementation on plasma NO levels. ${ }^{a, b, c}$ Mean values with unlike letters were significantly different $(P<0.05)$. There was a significant exercise $\times$ GSE interaction $(P=0.049)$. C, control; GC, GSE-supplemented control; CEC, chronic exercise control; GCE, GSE-supplemented chronic exercise; AEC, acute exercise control; GAE, GSE-supplemented acute exercise. 


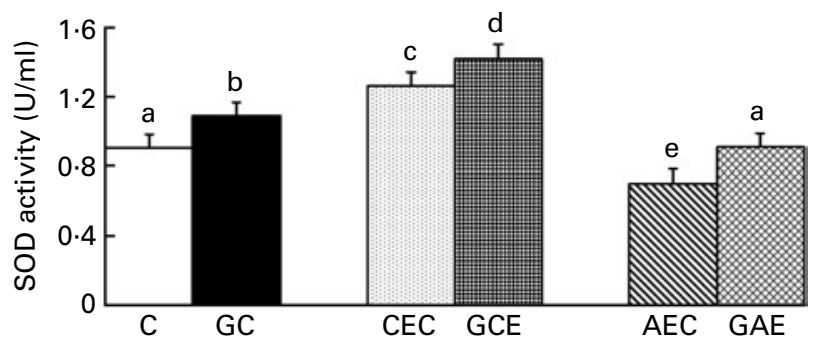

Fig. 5. Effects of acute or chronic exercise and grape seed extract (GSE) supplementation on plasma superoxide dismutase (SOD) activities. a,b,c,d,e Mean values with unlike letters were significantly different $(P<0.05$; two-way ANOVA). There was a significant exercise $\times$ GSE interaction $(P=0.032)$. C, control; GC, GSE-supplemented control; $\mathrm{CEC}$, chronic exercise control; GCE, GSE-supplemented chronic exercise; AEC, acute exercise control; GAE, GSE-supplemented acute exercise.

human subjects and rats ${ }^{(32,33)}$, and grape extract supplementation increased antioxidant capacity in athletes ${ }^{(15)}$. Based on these results, our findings supported the antioxidant potential of GSE against exercise-induced oxidative stress.

There are contradictory results regarding the changes of MDA levels measured as thiobarbituric acid-reactive substances in acute exhaustive exercise and chronic exercise training. Although it is generally demonstrated that MDA levels increased after acute exhaustive exercise in plasma, skeletal muscle, liver and lung tissues ${ }^{(1,25,34)}$, in several studies it has been suggested that MDA levels were not changed ${ }^{(35,36)}$ and even decreased ${ }^{(37)}$. For chronic exercise, consistent with our results, it has been demonstrated that regular exercise training increases resistance against lipid peroxidation and reduces oxidative protein and DNA damage ${ }^{(38)}$. The differences among the results can be explained by antioxidant nutritional status, exercise intensity, training level and methods used for the measurement of oxidative stress ${ }^{(6)}$. Chronic exercise can induce adaptations as a result of the cumulative effects of repeated bouts of sufficient intensity and duration that attenuate exercise-induced oxidative stress ${ }^{(39)}$. Therefore, the reduced oxidative stress resulting from chronic training may originate from the enhanced antioxidant defence system ${ }^{(39)}$. According to these results, our exercise training protocol seems to have enough intensity and duration to induce adaptation.

During exhaustive exercise, it has been reported that XO activity significantly increased in both blood and tissues ${ }^{(5,40)}$. Consistent with the previous studies, in the present study $\mathrm{XO}$ activity significantly increased after acute exhaustive exercise; however, chronic exercise did not affect XO activity. Furthermore, GSE supplementation decreased and therefore normalised XO activity to the control levels in acute exercised rats. The acute exhaustive exercise-induced increase in $\mathrm{XO}$ activity can be explained by acute exhaustive exerciseinduced ischaemia and/or hypoxia-like situations in the tissues. Ischaemia or hypoxia in certain regions of the body cause ATP to be converted to ADP, AMP, inosine and finally hypoxanthine. During ischaemia or hypoxia, oxygen concentrations are low and intracellular concentrations of $\mathrm{XO}$ and hypoxanthine can rise ${ }^{(41)}$. Our findings suggest that GSE supplementation inhibits the conversion of xanthine dehydrogenase to the XO or GSE supplementation improves the resistance of tissues against ischaemia. No study to date has investigated the effects of GSE supplementation on XO activity in exercised human subjects or rats. However, it has been demonstrated that GSE supplementation inhibits ischaemia-reperfusion injury-induced lipid peroxidation in the heart ${ }^{(20)}$ and kidney ${ }^{(42)}$. Because $\mathrm{XO}$ activity was not investigated in these studies, this is the originality of our study. In addition, unchanged XO activity in chronically exercised rats can be explained by the acute effects of exercise being removed in $24 \mathrm{~h}$ after exercise ${ }^{(41)}$.

In the present study, plasma ADA activity significantly increased after acute exhaustive exercise; however, chronic exercise did not affect ADA activity. GSE supplementation attenuated ADA activity in acute exercised rats to the control levels. It has been suggested that there is a positive correlation between MDA levels and ADA activity, while there is a negative correlation between antioxidant enzymes and ADA activity $^{(43)}$. In the present study, we showed a positive correlation between MDA levels and ADA activity $\left(R^{2} 0 \cdot 153\right.$, $P=0 \cdot 002)$. Consistent with our findings, Langfort et al. ${ }^{(44)}$ showed that ADA activity increased after exhaustive exercise in heart tissue of rats. It has been showed in several studies $^{(45,46)}$ that antioxidant supplementation decreased ADA activity. Based on these findings, our results supported the antioxidant potential of GSE. Unchanged ADA activity in chronically exercised rats can be explained by the acute effects of exercise being removed in $24 \mathrm{~h}$ after exercise ${ }^{(41)}$.

In our study, plasma NO levels was not affected by acute exhaustive exercise, while it was increased with both GSE supplementation and chronic exercise. It has been reported that $3,6,8$ and 12 months of exercise training increase plasma NO levels in rats ${ }^{(2,47)}$. During exercise training, increased NO is involved in the regulation of vasodilatation; hence it increases substrate supplies to muscles. Increased NO decreases superoxide production, and inhibits neutrophil aggregation and lysosomal enzyme release from neutrophils ${ }^{(48)}$. NO also has the capacity to inhibit XO to prevent cellular damage $^{(49)}$. In the present study, XO activity increased but NO level did not change in response to acute exercise.



Fig. 6. Effects of acute or chronic exercise and grape seed extract (GSE) supplementation on plasma glutathione peroxidase (GPx) activities. a,b,c Mean values with unlike letters were significantly different $(P<0.05$; twoway ANOVA). There was a significant exercise $\times$ GSE interaction $(P=0.040)$ C, control; GC, GSE-supplemented control; CEC, chronic exercise control; GCE, GSE-supplemented chronic exercise; AEC, acute exercise control; GAE, GSE-supplemented acute exercise. 
This can be explained by the compensatory role of NO against increased XO activity. It is generally demonstrated that GSE supplementation increases NO secretion ${ }^{(50,51)}$. Furthermore, Shao et al. $^{(52)}$ suggested that high-dose GSE may induce cytotoxicity by increasing oxidative stress caused by NO in cultured chick embryos and therefore increase apoptotic cell death. Based on the present findings, we suggest that GSE supplementation increases NO production and exercise training supports this increase.

In the present study, plasma antioxidant enzyme activities (SOD and GPx), while impaired after acute exhaustive exercise, improved after chronic exercise training and GSE supplementation increased SOD and GPx activities especially in both the exercised groups (acute and chronic). SOD is the major defence upon superoxide radicals and catalyses the dismutation of superoxide and the formation of $\mathrm{H}_{2} \mathrm{O}_{2}$. However, GPx has the ability to transform $\mathrm{H}_{2} \mathrm{O}_{2}$ into water ${ }^{(6)}$. Although there are contradictory results about the acute and chronic exercise-induced changes in the antioxidant enzyme activity, it has been generally accepted that both acute exhaustive exercise and chronic exercise training increase antioxidant enzyme activities in tissues such as skeletal muscle (2,53,54) $^{(2)}$ and erythrocytes ${ }^{(55,56)}$. It has been suggested that acute exercise-induced increase in antioxidant enzyme activation is due to the increased free-radical production during acute exhaustive exercise, while regular exercise training negates the harmful effects of oxidative stress by up-regulating the activity of antioxidant enzymes ${ }^{(57)}$. In the present study, the acute exhaustive exercise protocol might have induced a decrease in SOD and GPX activity because of the increased ROS production in tissues especially in the skeletal muscles. The observed decrease in antioxidant enzyme activity may reflect allosteric down-regulation of the enzymes in addition to enzyme inactivation attributable to overwhelming oxidative stress. In addition, Elosua et al. ${ }^{(58)}$ have shown that there is a transient decrease (at 30 min post exercise) in blood SOD and GPx activities, after a 30-min exercise bout. The authors postulate that exercise-induced ROS production leads to the consumption of enzyme activity with a subsequent rebound recovery. In the present study, we measured total SOD activity which includes all three types of SOD iso-enzymes $(\mathrm{Cu} / \mathrm{Zn}$, Mn and FeSOD).

Our findings demonstrated that 6 weeks of training protocol is good enough to stimulate antioxidant enzyme expression and synthesis. The difference in the results may depend on the difference in the analysis methods and intensity and duration of the training protocol. Our findings also suggest that GSE supplementation up-regulates antioxidant enzyme activity especially in acutely and chronically exercised rats.

In conclusion, while acute exhaustive exercise increases oxidative stress and therefore induces lipid peroxidation and an ischaemia-like situation, chronic exercise training in enough intensity and duration up-regulates antioxidant defence systems by increasing the antioxidant enzyme activity in acutely and chronically exercised rats. The present study demonstrated that GSE has strong antioxidant potential, attenuates exercise-induced oxidative damage and augments the activities of antioxidant enzymes; however, it does not affect physical performance. Since our study is the first investigation in this subject, more detailed further studies are needed to determine the mechanism of action and the antioxidant potential of GSE in exercise-induced oxidative stress.

\section{Acknowledgements}

The authors declare that there are no conflicts of interest. The authors thank Professor Said Bodur for the statistical assistance. This work was supported by Scientific Research and Project Coordinator of Selcuk University (grant no. SABE 08102008). All the authors contributed to the collection and interpretation of the data as well as to the drafting of the manuscript.

\section{References}

1. Davies KJ, Quintanilha AT, Brooks GA, et al. (1982) Free radicals and tissue damage produced by exercise. Biochem Biophys Res Commun 107, 1198-1205.

2. Husain $\mathrm{K}$ (2003) Interaction of exercise training and chronic NOS inhibition on blood pressure, heart rate, NO and antioxidants in plasma of rats. Pathophysiology 10, 47-56.

3. Osorio RA, Christofani JS, D'Almeida V, et al. (2003) Reactive oxygen species in pregnant rats: effects of exercise and thermal stress. Comp Biochem Physiol C Toxicol Pharmacol 135, 89-95.

4. Alessio HM, Goldfarb AH \& Cutler RG (1988) MDA content increases in fast- and slow-twitch skeletal muscle with intensity of exercise in a rat. Am J Physiol 255, 874-877.

5. Radák Z, Asano K, Inoue M, et al. (1995) Superoxide dismutase derivative reduces oxidative damage in skeletal muscle of rats during exhaustive exercise. J Appl Physiol 79, $129-135$.

6. Finaud J, Lac G \& Filaire E (2006) Oxidative stress: relationship with exercise and training. Sports Med 36, 327-358.

7. Yu BP (1994) Cellular defenses against damage from reactive oxygen species. Physiol Rev 74, 139-162.

8. Gomez-Cabrera MC, Domenech E \& Viña J (2008) Moderate exercise is an antioxidant: upregulation of antioxidant genes by training. Free Radic Biol Med 44, 126-131.

9. Banerjee AK, Mandal A, Chanda D, et al. (2003) Oxidant, antioxidant and physical exercise. Mol Cell Biochem 253, 307-312.

10. Khanna S, Atalay M, Laaksonen DE, et al. (1999) Alpha-lipoic acid supplementation: tissue glutathione homeostasis at rest and after exercise. J Appl Physiol 86, 1191-1196.

11. Atalay M, Laaksonen DE, Khanna S, et al. (2000) Vitamin E regulates changes in tissue antioxidants induced by fish oil and acute exercise. Med Sci Sports Exerc 32, 601-607.

12. Kar P, Laight D, Shaw KM, et al. (2006) Flavonoid-rich grape seed extracts: a new approach in high cardiovascular risk patients? Int J Clin Pract 60, 1484-1492.

13. Bagchi D, Garg A, Krohn RL, et al. (1997) Oxygen free radical scavenging abilities of vitamins $\mathrm{C}$ and $\mathrm{E}$, and a grape seed proanthocyanidin extract in vitro. Res Commun Mol Pathol Pharmacol 95, 179-189.

14. Minegishi Y, Haramizu S, Hase T, et al. (2011) Red grape leaf extract improves endurance capacity by facilitating fatty acid utilization in skeletal muscle in mice. Eur J Appl Physiol 111, 1983-1989. 
15. Lafay S, Jan C, Nardon K, et al. (2009) Grape extract improves antioxidant status and physical performance in elite male athletes. J Sport Sci Med 8, 468-480.

16. Veskoukis AS, Nikolaidis MG, Kyparos A, et al. (2009) Blood reflects tissue oxidative stress depending on biomarker and tissue studied. Free Radic Biol Med 47, 1371-1374.

17. Bagchi D, Garg A, Krohn RL, et al. (1998) Protective effects of grape seed proanthocyanidins and selected antioxidants against TPA-induced hepatic and brain lipid peroxidation and DNA fragmentation, and peritoneal macrophage activation in mice. Gen Pharmacol 30, 771-776.

18. Joshi SS, Kuszynski CA, Bagchi M, et al. (2000) Chemopreventive effects of grape seed proanthocyanidin extract on Chang liver cells. Toxicology 155, 83-90.

19. Feng Y, Liu YM, Fratkins JD, et al. (2005) Grape seed extract suppresses lipid peroxidation and reduces hypoxic ischemic brain injury in neonatal rats. Brain Res Bull 66, 120-127.

20. Sato M, Maulik G, Ray PS, et al. (1999) Cardioprotective effects of grape seed proanthocyanidin against ischemic reperfusion injury. J Mol Cell Cardiol 31, 1289-1297.

21. Sehirli O, Ozel Y, Dulundu E, et al. (2008) Grape seed extract treatment reduces hepatic ischemia-reperfusion injury in rats. Phytother Res 22, 43-48.

22. Sen CK \& Bagchi D (2001) Regulation of inducible adhesion molecule expression in human endothelial cells by grape seed proanthocyanidin extract. Mol Cell Biochem 216, 1-7.

23. El-Alfy AT, Ahmed AA \& Fatani AJ (2005) Protective effect of red grape seeds proanthocyanidins against induction of diabetes by alloxan in rats. Pharmacol Res 52, 264-270.

24. Balu M, Sangeetha P, Murali G, et al. (2006) Modulatory role of grape seed extract on age-related oxidative DNA damage in central nervous system of rats. Brain Res Bull $\mathbf{6 8}$, 469-473.

25. Liu J, Yeo HC, Overvik-Douki E, et al. (2000) Chronically and acutely exercised rats: biomarkers of oxidative stress and endogenous antioxidants. J Appl Physiol 89, 21-28.

26. Gul M, Laaksonen DE, Atalay M, et al. (2002) Effects of endurance training on tissue glutathione homeostasis and lipid peroxidation in streptozotocin-induced diabetic rats. Scand J Med Sci Sports 12, 163-170.

27. Wasowicz W, Nève J \& Peretz A (1993) Optimized steps in fluorometric determination of thiobarbituric acid-reactive substances in serum: importance of extraction $\mathrm{pH}$ and influence of sample preservation and storage. Clin Chem 39, $2522-2526$

28. Prajda N \& Weber G (1975) Malignant transformation-linked imbalace: decreased XO activity in hepatomas. FEBS Lett $\mathbf{5 9}$, 245-249.

29. Giusti G (1974) Adenosine deaminase. In Methods of Enzymatic Analysis, 2nd ed., pp. 1092-1098 [MV Bergmeyer, editor]. New York, NY: Academic Press.

30. Cortas NK \& Wakid NW (1990) Determination of inorganic nitrate in serum and urine by a kinetic cadmium-reduction method. Clin Chem 36, 1440-1443.

31. Enginar H, Cemek M, Karaca T, et al. (2007) Effect of grape seed extract on lipid peroxidation, antioxidant activity and peripheral blood lymphocytes in rats exposed to x-radiation. Phytother Res 21, 1029-1035.

32. Alessio HM, Hagerman AE, Romanello M, et al. (2002) Consumption of green tea protects rats from exercise-induced oxidative stress in kidney and liver. Nutr Res 22, 1177-1188.

33. Panza VS, Wazlawik E, Ricardo Schütz G, et al. (2008) Consumption of green tea favorably affects oxidative stress markers in weight-trained men. Nutrition 24, 433-442.

34. Lin WT, Yang SC, Chen KT, et al. (2005) Protective effects of L-arginine on pulmonary oxidative stress and antioxidant defenses during exhaustive exercise in rats. Acta Pharmacol Sin 26, 992-999.

35. Child RB, Wilkinson DM \& Fallowfield JL (1999) Resting serum antioxidant status is positively correlated with peak oxygen uptake in endurance trained runners. $J$ Sports Med Phys Fitness 39, 282-284.

36. Dixon CB, Robertson RJ, Goss FL, et al. (2003) Effect of resistance training status on free radical production and muscle damage following acute exercise. Med Sci Sports Exerc 35, 157.

37. Kretzschmar M, Müller D, Hübscher J, et al. (1991) Influence of aging, training and acute physical exercise on plasma glutathione and lipid peroxides in man. Int J Sports Med 12, $218-222$

38. Leeuwenburgh C \& Ji LL (1998) Glutathione and glutathione ethyl ester supplementation of mice alter glutathione homeostasis during exercise. J Nutr 128, 2420-2426.

39. Bloomer RJ \& Goldfarb AH (2004) Anaerobic exercise and oxidative stress: a review. Can J Appl Physiol 29, 245-263.

40. Liu CC, Huang CC, Lin WT, et al. (2005) Lycopene supplementation attenuated xanthine oxidase and myeloperoxidase activities in skeletal muscle tissues of rats after exhaustive exercise. Br J Nutr 94, 595-601.

41. Sachdev S \& Davies KJ (2008) Production, detection, and adaptive responses to free radicals in exercise. Free Radic Biol Med 44, 215-223.

42. Nakagawa T, Yokozawa T, Satoh A, et al. (2005) Attenuation of renal ischemia-reperfusion injury by proanthocyanidinrich extract from grape seeds. J Nutr Sci Vitaminol (Tokyo) 51, 283-286.

43. Erkiliç K, Evereklioglu C, Cekmen M, et al. (2003) Adenosine deaminase enzyme activity is increased and negatively correlates with catalase, superoxide dismutase and glutathione peroxidase in patients with Behçet's disease: original contributions/clinical and laboratory investigations. Mediators Inflamm 12, 107-116.

44. Langfort J, Czarnowski D, Pilis W, et al. (1996) Effect of various types of exercise training on $5^{\prime}$-nucleotidase and adenosine deaminase activities in rat heart: Influence of a single bout of endurance exercise. Biochem Mol Med 59, 28-32.

45. Bassini-Cameron A, Sweet E, Bottino A, et al. (2007) Effect of caffeine supplementation on haematological and biochemical variables in elite soccer players under physical stress conditions. Br J Sports Med 41, 523-530.

46. Ozyurt B, Iraz M, Koca K, et al. (2006) Protective effects of caffeic acid phenethyl ester on skeletal muscle ischemiareperfusion injury in rats. Mol Cell Biochem 292, 197-203.

47. Xiao DS \& Qian ZM (2000) Plasma nitric oxide and iron concentrations in exercised rats are negatively correlated. Mol Cell Biochem 208, 163-166.

48. Adams HR (1996) Physiologic, pathophysiologic, and therapeutic implications for endogenous nitric oxide. J Am Vet Med Assoc 209, 1297-1302.

49. Souza-Costa DC, Zerbini T, Metzger IF, et al. (2005) L-Arginine attenuates acute pulmonary embolism-induced oxidative stress and pulmonary hypertension. Nitric Oxide 12, 9-14.

50. Demrow HS, Slane PR \& Folts JD (1995) Administration of wine and grape juice inhibits in vivo platelet activity and thrombosis in stenosed canine coronary arteries. Circulation 91, 1182-1188.

51. Clifton PM (2004) Effect of grape seed extract and quercetin on cardiovascular and endothelial parameters in high-risk subjects. J Biomed Biotechnol 2004, 272-278. 
52. Shao ZH, Hsu CW, Chang WT, et al. (2006) Cytotoxicity induced by grape seed proanthocyanidins: role of nitric oxide. Cell Biol Toxicol 22, 149-158.

53. Ji LL \& Fu R (1992) Responses of glutathione system and antioxidant enzymes to exhaustive exercise and hydroperoxide. J Appl Physiol 72, 549-554.

54. Leeuwenburgh C, Hollander J, Leichtweis S, et al. (1997) Adaptations of glutathione antioxidant system to endurance training are tissue and muscle fiber specific. Am J Physiol 272, 363-369.

55. Ohno H, Yahata T, Sato Y, et al. (1988) Physical training and fasting erythrocyte activities of free radical scavenging enzyme systems in sedentary men. Eur J Appl Physiol Occup Physiol 57, 173-176.
56. Marzatico F, Pansarasa O, Bertorelli L, et al. (1997) Blood free radical antioxidant enzymes and lipid peroxides following long-distance and lactacidemic performances in highly trained aerobic and sprint athletes. J Sports Med Phys Fitness 37, 235-239.

57. Greathouse KL, Samuels M, Dimarco NM, et al. (2005) Effects of increased dietary fat and exercise on skeletal muscle lipid peroxidation and antioxidant capacity in male rats. Eur J Nutr 44, 429-435.

58. Elosua R, Molina L, Fito M, et al. (2003) Response of oxidative stress biomarkers to a 16-week aerobic physical activity program, and to acute physical activity, in healthy young men and women. Atherosclerosis 167, $327-334$. 